Fecha de recepción: marzo 2011 Fecha de aceptación: junio 2011 Versión final: septiembre 2012

\section{Industrias Creativas de Diseño de Indumentaria de Autor. Diagnóstico y desafíos a 10 años del} surgimiento del fenómeno en Argentina

Laureano Mon *

\begin{abstract}
Resumen: Un diagnóstico productivo y cultural de las Industrias Creativas de Diseño de Indumentaria de Autor en Argentina, a 10 años de su surgimiento, nos permitirá medir el real impacto de este sector a la vez que identificar los principales desafíos para lograr la sustentabilidad económica y social en el largo plazo.
\end{abstract}

Palabras clave: creatividad - cultura - desarrollo local - diagnóstico productivo - Diseño - educación - Indumentaria de Autor - industria - políticas públicas - sustentabilidad.

[Resúmenes en inglés y portugués en la página 34]

${ }^{(*)}$ Licenciado en Ciencia Política por la Facultad de Ciencias Sociales de la UBA. Se desempeña actualmente en áreas de gestión, diseño e implementación de programas de apoyo y capacitación para la industria de la indumentaria y textil para el Observatorio de Tendencias del Instituto Nacional de Tecnología Industrial (INTI).

\title{
Introducción
}

La industria de la indumentaria en Argentina ha comenzado a vivenciar un gran desarrollo creativo en los últimos 10 años a causa del surgimiento de una masa crítica de diseñadores que, al frente de sus propias empresas, han redefinido la concepción e implicancias del vestir. Estos nuevos referentes conforman lo que comúnmente se conoce como el diseño de autor, una denominación que se presta a confusión y polémica -se suele sostener que todo el diseño es de autor, una discusión que excede a este artículo- pero que sin embargo es útil para el lector en general que suele identificar sin problemas el tipo de productos del cual hablamos. Nombres como Martín Churba (Tramando), Cora Groppo, Mariano Toledo, Vero Ivaldi, Pablo Ramírez, Jessica Trosman, Javier y Alejo Estebecorena (HE), Mariana Dappiano, Araceli Pourcel, Rosa y Juana Benedit (Benedit Bis), Nadine Zlotogora, Vicki Otero, Mariana Cortes (Juana de Arco), Mario Buraglio y Victor Delgrosso (Varanasi), Verónica Fiorini y Anabel Wichmacki, Leandro Domínguez, Min Agostini, José Luis Otiñiano (Oxdans), Rodrigo Abarquero y Débora Di Stillo (Objeto), Camila Milesi y Emiliano Blanco (Kostume), Valeria Pesqueira (Pesqueira TM), Cecilia Gadea, Marcelo Senra, Florencia Fiocca, y Manuela Rasjido, entre otros, formaron parte de una primer grupo de diseñadores que, a comienzos de la década, abrieron caminos para 
una industria creativa del diseño de indumentaria basada en la originalidad e innovación. El desmarcamiento respecto del discurso de la moda masiva implicó asumir riesgos importantes en términos económicos y culturales, por ello la coincidencia en el tiempo del comienzo de esas empresas como así también su continuidad actual es un dato fundamental en nuestro análisis. A ello hay que sumar los nuevos emprendimientos de diseñadores que comenzaron luego su actividad en todo el país y que hoy también conforman el segmento como Roxana Liendro (Santos Liendro), Jimena Anastasio, German Lang (Langg), Nicolás Silva Abriola y Mariana Fernández Barrios (Y es Agua), Victoria Bessone (La Primitiva), Silvia Querede y Noelí Gómez (Quier), Débora Orellana (Pequeños Trazos), Marina Gryciuk, Leo Peralta, Carolina Yrigaray, Caro Sosa, Ale Gougy (Cosecha), Juan Manuel Alzamora (Vida de Perros), Carolina Bloise (Crista), Carola Bessaso (DAM), Eloísa López (Audia Valdez), Ezequiel y Viviana Toledo (EzequielT), Florencia Cacciabue (Florece), Lucila Negri, Josefina Luna, Laura Scrimini, Fabiola Brandan, Lorena Sosa, Cristina Pérez (Ornato y Menaje), Paula Ledesma, Raquel Rosetto, María Spina, Augusta Peterle, Viviana Uchitel, Gabi Candioti, Verónica Di Miero (Seco), Clara de la Torre y Diana Chai (Manto), Dorina Vidoni, Lisandro Cocorda (Lisobono), Carlos Bono, Agustín Bossini Pithod, Paola Balcaza y Samir Nait (NSBP), Paula Yaculb y Leonel Halpern (Colombas), Miryam Millán, Mercedes Martínez y Mariano Breccia (Ropa Doceñada), entre otros.

Actualmente, el diseño de autor produce bienes que se insertan en un mercado de consumo donde los productos de moda dominan ampliamente -identificado por otros autores como también pertenecientes a las industrias creativas, lo cual implicaría analizar hasta que punto hacer productos bajo las tendencias de moda en un país desfasado en temporada es un hecho original- sin embargo la lógica de las piezas de autor se basa en la búsqueda de identificación con el usuario a otro nivel. Al ser bienes concebidos y producidos para diferenciarse del resto de la oferta a partir de una gran cuota de creatividad, privilegiando una visión personal de quien los diseña, se convierten en prendas con una carga identitaria más fuerte, portadoras de valores, que nos hablan de un contexto social bajo el cual fue producido y de una cultura común de trabajo dentro de la cual se insertan. Tanto los bienes en sí con su trazabilidad implican un gran valor simbólico. No debe ser un dato menor la originalidad e innovación que estos lenguajes de diseño entrañan, abriéndose paso y asumiendo riesgos, frente a una dinámica de consumo que tiende a la homogeneización.

El objetivo de éste artículo es aportar elementos que nos permitan profundizar en el estado actual de la Industria Creativa de Diseño de Indumentaria de Autor en Argentina, analizando brevemente sus implicancias tanto productivas como culturales, así también dinámicas y proyecciones de crecimiento. Para ello me valdré de información producida por el Observatorio de Tendencias del Instituto Nacional de Tecnología Industrial (INTI) y Fundación Pro Tejer, en relación a éste segmento particular de la industria. Para aproximarme a las implicancias económicas tomaré indicadores elaborados por la Encuesta Económica Nacional de Diseño Indumentaria de Autor 2010 (INTI, 2010) y asimismo citaré algunas reflexiones escritas para dicho estudio el cual tuve la dicha de co-dirigir. La investigación Mapa de Diseño Argentino (INTI, 2007-10) será de gran ayuda para obtener algunos lineamientos acerca de las implicancia culturales del segmento, como así también la fuente de información de Por La Calle, Circuitos de Diseño (INTI, 2008-10) nos aportará elementos contextuales locales. 


\section{Devenir del diseño de indumentaria de autor}

Todo fenómeno ocurrido en la última década en nuestro país implica un doble desafío: por un lado, tratar con la novedad del fenómeno mismo repercutiendo en la observación del objeto de estudio, y, por otro lado, el carácter inadecuado -en este caso- de las herramientas de análisis a partir de las cuales realizar una aproximación para poder dilucidar elementos que faciliten su comprensión. Este último punto adquiere relevancia a partir de dimensionar la caída de paradigmas sociales, culturales y económicos ocasionada por la crisis de 2001. La crítica y cuestionamiento a conceptos del discurso hasta ese entonces dominante ha sido muy fuerte, por lo que fenómenos emergentes de esa situación plantearon formas novedosas de creación y trabajo para nuestro país. Responder a situaciones que se revelaron contra lo establecido con herramientas tradicionales en una sociedad altamente dinámica es algo para replantearse de antemano.

El diseño de indumentaria de autor es tributario de esa crisis y nació en el gesto inicial de sobrevivencia. Gran parte de los diseñadores -que más tarde se convertirían en una primera ola de referentes del diseño nacional- no tenían nada que perder porque estaban recientemente desocupados y necesitaban sobrevivir en un contexto dominado por la incertidumbre. En este sentido son recurrentes las historias personales que narran cómo con la indemnización proporcionada por las grandes empresas que los despidieron los diseñadores compraron sus primeras piezas textiles para producir sin siquiera tener un plan creativo o lenguaje de diseño definido previamente. No había tiempo para armar planes de negocios ni planificar los procesos de diseño y producción. Había que hacer. Alguna vez en un café de San Telmo, Ana Gertner (2008), una artista plástica también diseñadora, me proporcionó la clave para comprender el espíritu de la creación en nuestro país: "el diseñador argentino está más preocupado por el hacer que por el lenguaje", me dijo con una sonrisa. Y es cierto, tal vez ese patrón se repita en distintos aspectos del ser nacional.

Los diseñadores comenzaron a crear, producir y comercializar primeramente como una forma de autoempleo capaz de brindarles una mejor calidad de vida, para luego fundar sus empresas de diseño a través de un proceso de formalización. Esto que sucedió en el ámbito de la indumentaria también ocurrió en otras disciplinas del diseño como el calzado, bijouterie, marroquinería y objetos. Es probable que de todos los ámbitos del diseño sea el de objetos industriales el que contase con mayor tradición ya que las primeras universidades con carreras de diseño industrial se fundaron a mediados del Siglo XX -en la Universidad Nacional de Cuyo, Mendoza, se abrió en 1958 la primera formación académica en esta disciplina en Latinoamérica- mientras que Indumentaria se abrió por primera vez en el país en 1988 en la Universidad de Buenos Aires.

Analizar el trabajo de estos diseñadores y de sus empresas de indumentaria implica acercarnos a un hecho desde dos perspectivas: económica y cultural. Desde la primera de ellas, las firmas adquieren importancia en tanto emprendimientos productivos con distintos grados de desarrollo, desde micro emprendimientos hasta PYMES, y donde entran en juego variables como producción, empleo, inversión, recursos, capital, financiación, comercialización y capacitación. La dimensión cultural entra en escena desde el momento en que estos diseñadores desarrollan un ejercicio creativo logrando, con el tiempo, un lenguaje de diseño propio que utiliza un criterio independiente a la hora de proponer productos originales en los mercados nacionales e internacionales. Todo proceso creativo personal se inscribe dentro de un contexto cultural y social que lo contiene. 


\section{Perfil de las empresas y productos de diseño de indumentaria de autor}

Dentro de la cadena de la Agro Industria de Indumentaria y Textil, podemos incluir en la denominación diseño de indumentaria de autor a aquellas empresas que diseñan y producen prendas con alto valor agregado a partir de procesos de trabajo intensivos que incorporan importantes dosis de creatividad y experimentación en uno o más aspectos del producto, como ser la morfología y/o textura y/o tipología y/o simbología de las piezas. Por ende, la producción y comercialización de estos bienes diferenciales genera no sólo el desarrollo económico sino que también garantiza la diversidad cultural de una comunidad. En indumentaria, el criterio de originalidad se evidencia en productos que logran escapar a las tendencias de moda masivas a través de lenguajes creativos innovadores sostenidos en el tiempo.

De acuerdo al Mapa de Diseño Argentino (INTI, 2007-10), sabemos que en nuestro país hay al menos 150 empresas que producen éste tipo de productos de manera estable -con una antigüedad mínima en el mercado de 1 año-, siendo ésta la población objetiva que debiéramos considerar para obtener indicadores viables para nuestras reflexiones. Es cierto que contabilizando aquellas firmas de indumentaria de autor que dan sus primeros pasos (menos de 1 año), caracterizadas por la informalidad e inestabilidad en la producción y comercialización, el número de población se podría incrementar considerablemente, sin embargo aportaría elementos endebles para nuestro fin.

El diseño de indumentaria de autor es un segmento novedoso en el mercado nacional que se ha desarrollado con fuerza en los últimos 10 años. Por ende, se trata de micro y pequeñas empresas jóvenes -la gran mayoría no supera la década de antigüedad-, llevadas adelante -en gran medida- por diseñadores sin experiencia previa en la gestión empresarial.

En este sentido, es significativo el carácter joven y dinámico de los emprendimientos tal como habíamos señalado anteriormente. Al observar la antigüedad de las empresas queda en evidencia el punto de inflexión que significó la crisis del 2001 para la actividad y, por ello, lo reciente del fenómeno del diseño. Antes de ese año, sólo el $15 \%$ de los emprendimientos ya producía y comercializaba. Luego de 2001, surgieron el $85 \%$ de las empresas de diseño de autor que conocemos actualmente. Yendo un poco más en profundidad, entre el 2001 y el 2003, el $25 \%$ de los emprendimientos comenzaron su actividad. Entre el 2004 y el 2006, se sumaron $33 \%$ más, siendo el período de mayor crecimiento coincidente con el comienzo del ciclo ascendente de la economía argentina; para, finalmente, en los últimos 3 años, sumarse un 27\% más de firmas de diseño de autor al mercado nacional (INTI, 2010).

Los profesionales que llamamos diseñadores de indumentaria de autor y que producen prendas -bajo ese criterio- a partir de sus propias empresas tienen en su origen una formación heterogénea, a nivel de disciplinas y fuentes de aprendizaje, pudiendo encontrar en éste último caso tanto egresados de universidades como autodidactas. Si bien las características culturales y educativas de cada ciudad influyen en el perfil de los profesionales -por ejemplo en Córdoba la presencia del diseño gráfico es importante, al igual que en Mendoza el diseño industrial, en Buenos Aires la arquitectura, y en Tucumán las carreras de diseño de interiores, mientras que en Rosario (Santa Fe) las Bellas Artes son claves para comprender el panorama- las formaciones de origen están relacionadas con actividades creativas siendo, en muchos casos, lo más cercano que las personas encuentran en regiones donde la carrera de diseño de indumentaria es reciente.

La relevancia de estos datos contextuales sirve para comprender por qué sólo el 34\% de los 
diseñadores tiene una formación académica vinculada específicamente al diseño de indumentaria y textil, considerando en éste grupo tanto a aquellos que estudiaron carreras universitarias como terciarias. Otro grupo lo conforman aquellos que tienen como formación de origen otras carreras del área del diseño, totalizando un $15 \%$, donde el Diseño Gráfico lidera con un $12 \%$, siguiendo el Diseño Industrial con un $3 \%$. Los arquitectos devenidos en diseñadores suman un $7 \%$, así como aquellos que se formaron en Diseño de Interiores representan un $4 \%$. Sumando las formaciones académicas y autodidactas vinculadas al Arte, tenemos un $22 \%$, siendo un 9 $\%$ aquellos que estudiaron académicamente Bellas Artes; un $7 \%$ quienes provienen de las artes plásticas de manera autodidacta; un $6 \%$ de artistas de otras disciplinas también formados de manera autodidacta; y un $1 \%$ artistas audiovisuales formados en universidades. Los artesanos que actualmente producen prendas de diseño contemporáneo representan un $4 \%$. Finalmente, los diseñadores provenientes del mundo de la publicidad son un $3 \%$ (INTI, 2010).

Sobre ésta formación heterogénea de los recursos humanos -donde solo un tercio de los diseñadores estudiaron Diseño de Indumentaria y Textil- es sobre la cual podemos encontrar una de las razones de la diversidad de los lenguajes creativos del diseño de autor, donde la experimentalidad en base a cruce de competencias y recursos genera respuestas inéditas para crear productos innovadores. La riqueza cultural de ésta industria creativa comienza con ésta libertad para realizar fusiones entre el arte, el diseño y la artesanía, en bienes técnicamente híbridos con perfiles estéticos contemporáneos.

Asimismo, esta heterogeneidad que posibilita grandes cuotas de creatividad explica, en gran medida, las falencias que muchas veces encontramos en términos de calidad -terminación, moldería, texturas- en algunos desarrollos iniciales. Nada que no se pueda corregir con capacitaciones adecuadas. Pero también las carencias en la gestión de diseño repercuten en la imposibilidad de establecer, en muchos casos, correctas planificaciones y estrategias de producción. Esto cuenta, de igual modo, para aquellos que recibieron formación académica en indumentaria y textil dentro de carreras jóvenes con un diseño curricular aún no dirigido a formar diseñadores con conocimientos productivos (ya sea para trabajar dentro de empresas de terceros como para fundar sus propias firmas). Estas observaciones impiden un mayor entendimiento de las posibles articulaciones que un diseñador puede realizar con la cadena industrial optimizando recursos y esfuerzos.

En contrapartida, firmas como Tramando, Trosman, Juana de Arco, Mariano Toledo, Mariana Dappiano, Hermanos Estebecorena, Varanasi y Cora Groppo, sólo por nombrar algunas, son ejemplos de empresas consolidadas y exitosas -desde el punto de vista organizativo, productivo y comercial- que marcan el rumbo en el mercado nacional estableciendo paradigmas de acción factibles para los nuevos emprendimientos.

La necesidad de contextualizar las empresas para comprender la naturaleza de su actividad, nos obliga a ver su distribución territorial en el mapa pudiendo no sólo localizarlas y mensurarlas, sino también relacionarlas a un desarrollo local vinculado al diseño. Respecto de la ubicación geográfica de las empresas se destaca una gran concentración en la ciudad de Buenos Aires, representando el $58 \%$ de la producción nacional; seguida por Córdoba 12\% y Rosario (Santa Fe) $11 \%$. Mendoza $6 \%$ y S. M de Tucumán 5\%, se encuentran en una tercera línea entre los conglomerados urbanos con más presencia de emprendimientos de diseño. Lejos se ubican Gran Buenos Aires 2\%, y luego el resto de las provincias con un $1 \%$ (INTI, 2010).

El desarrollo de las industrias creativas de diseño de indumentaria está asociado directamente a 
un conjunto de variables que deben desenvolverse, al menos la mayoría de ellas, conjuntamente. Entre las principales condiciones para que se desarrolle una industria creativa de diseño de autor podemos encontrar:

- Instituciones educativas - públicas y/o privados- que fomenten las disciplinas del diseño en general, y el diseño de indumentaria en particular. Asimismo, los enfoques de los programas curriculares y los docentes de las carreras también ejercen su influencia condicionando en gran medida las expectativas de trabajo de los futuros diseñadores.

- Un mercado de indumentaria con canales de difusión y distribución que posibiliten desarrollar el segmento de autor; como ser tiendas, ferias y exposiciones, que conformen circuitos de diseño accesibles a los interesados en consumir estos productos.

- Público consumidor, local y/o extranjero, de diseño de autor. Esta variable muchas veces se encuentra inicialmente asociada a segmentos jóvenes receptivos de propuestas culturales alternativas, que encuentra su mayor expansión cuando públicos medios -a nivel etario y socio económico- comienzan a incorporar en sus hábitos de consumo estos bienes.

- Medios masivos de comunicación -tradicionales y/o alternativos- que den cuenta de las nuevas producciones de diseño; como ser diarios, revistas, radios, TV, plataformas digitales y redes sociales; colaborando en poner de moda al diseño.

- Actores institucionales -públicos y/o privados- que desarrollen acciones de apoyo económico y cultural destinadas a emprendimientos de diseño. Dentro de este grupo podemos encontrar instituciones con distintos enfoques (culturales, turísticos, productivos, sociales) que coinciden en fomentar estas Industrias Creativas.

- Oferta de proveedores de bienes y servicios que complementen la producción de las empresas de diseño de indumentaria de autor. Esto significa, principalmente, que los diseñadores al frente de sus emprendimientos puedan acceder a materiales y servicios productivos de acuerdo a su escala y contemplando su objetivo de diferenciación.

- Referentes locales del diseño con desarrollos exitosos, donde los nuevos diseñadores se puedan ver reflejados y motivados en sus carreras profesionales.

Teniendo en cuenta estas variables es que podemos comprender el alto grado de desarrollo de la ciudad de Buenos Aires como epicentro nacional del diseño de indumentaria de autor, seguido luego por otros grandes centros urbanos como Córdoba, Rosario, Mendoza y San Miguel de Tucumán. Independientemente de su ubicación geográfica hay un patrón que se repite en las labores de los diseñadores que se encuentran al frente de sus firmas: la escasa división de tareas. La presencia de diseñador, convertido también en empresario, se da en todos los puntos del proceso de generación de una prenda comenzando por la concepción y diseño, pasando por las distintas fases del proceso productivo, hasta llegar a la difusión y comercialización de la misma. La intervención constante del diseñador/empresario permite que su sello esté presente en todas 
las fases de la empresa pero, a la vez, genera una recarga de su tarea que repercute en forma negativa en la organización general del proceso. El agotamiento se incrementa si ésta constante sucede no porque el diseñador tenga inconvenientes para delegar parte de sus funciones sino por la falta de recursos para poder contratar personal calificado para esas asignaturas. Muchas veces las falencias en la gestión y planificación generan inestabilidades en la producción de los bienes logrando altibajos en la oferta.

En cuanto a la producción en sí de las prendas es notable la gran integración de instancias industriales y artesanales que se da en diversas etapas del proceso. El trabajo intensivo se aplica principalmente en detalles que acentúan la diferenciación de los productos aumentando su valor agregado. Asimismo, la innovación generada a partir de pequeños o grandes cambios en el hacer, que tiene como elemento no conflictivo la prueba y error a través de un mix de estrategias tradicionales y alternativas, reactualiza técnicas y recursos constantemente, logrando un proceso dinámico de innovación. Esta cultura común de trabajo que aúna los desarrollos de los diseñadores a lo largo de todo el país, explica la heterogeneidad de lenguajes creativos que podemos observar cuando recorremos las producciones.

Del mismo modo, ésta creatividad es directamente proporcional a las limitaciones que deben enfrentar las empresas de diseño de autor en cuanto al acceso de bienes y servicios productivos. En este sentido, la escasez en la oferta de textiles para estos emprendimientos-que por la demanda de bajos volúmenes quedan fuera de la variedad y costos a los que puede acceder una gran empresa de moda- es fundamental para comprender ésta búsqueda constante de innovación -a partir de materiales comunes a todos- aplicada a texturas, morfología, tipologías y/o imaginarios.

Los resultados de la investigación Mapa de Diseño Argentino develaron estas estrategias de trabajo comunes a todos los diseñadores. Este diagnóstico inicial fue confirmado a través de la experiencia Las Cosas del Quehacer, debates en torno al diseño de indumentaria en Argentina realizada en la ciudad de Córdoba en mayo de 2010 por el Centro Cultural España Córdoba, INTI y Fundación Pro Tejer; donde 25 diseñadores de distintas partes del país -Misiones, Chaco, Salta, Jujuy, Córdoba, Santa Fe, Mendoza, Tucumán y ciudad de Buenos Aires- participaron en instancias colectivas de reflexión alrededor de temáticas como identidad, derechos de autor y sustentabilidad económica del diseño de autor.

De ésta experiencia surgieron conclusiones valiosas donde los mismos diseñadores afirmaban que su creatividad está asociada a la realidad socio económica bajo la cual desarrollan su actividad siendo la limitante de los materiales un motor inicial de creación. Asimismo reconocían que no hay una estética nacional identificable, como sí la tienen otros países, sino una comunión en los rasgos productivos lo cual significa que "existen puntos de contacto entre las distintas soluciones que cada diseñador emprende para resolver problemas comunes a todos" (CCEC, 2010). Más allá de la interacción con el entorno económico y social -inevitable para cualquier actividad productiva- la creación, consciente e inconsciente, de prendas que dialogan con los contextos geográficos y/o simbólicos también es un rasgo importante. La territorialidad de los lenguajes de diseño es evidente, lo que acentúa la trazabilidad de las prendas contemporáneas incrementando su valor conceptual que adquiere significado en un tiempo y espacio identificable. Esa marcada gestualidad de los productos permite que trasciendan la dinámica efímera de la moda colaborando en un diseño que busca perdurar en el vestuario de los consumidores acompañando sus historias de vida.

La relación con la cultura -imaginarios, geografía, símbolos, técnica, labores, materiales, tradi- 
ciones- genera un permanente trabajo cotidiano de reactualización del patrimonio intangible de la comunidad. El diseño nacional valoriza - casi sin pretensiones- la manera de ser y estar en el mundo del ser argentino, contribuyendo a la construcción de la identidad como un proceso dinámico y colectivo. Recorrer las producciones de Santos Liendro retomando el trabajo de la orfebrería en alpaca aplicada a prendas de modal; La Primitiva revalorizando el uso del aguayo y la cerámica en prendas de denim; Araceli Pourcel y su experimentación con los tejidos a dos agujas; Marcelo Senra y la aplicación de chaguar a vestidos; Manto con su reinterpretación del telar andino; Miryam Millán y las técnicas de pintura con parafina sobre seda; Juana de Arco y el recurso del ñandutí en prendas urbanas; Cecilia Gadea y el trabajo de bordado; sólo por mencionar unos pocos, son ejemplo de cómo labores ancestrales están presentes en los bienes actuales. Estas características no son exclusivas de la indumentaria ya que estas mismas empresas complementan la producción de prendas con otros rubros como calzado, marroquinería, accesorios y objetos, logrando ampliar las familias de productos. Esta inteligente estrategia permite no sólo ampliar la oferta de bienes para el público habitual sino que permite captar consumidores potenciales no habituados al consumo de indumentaria de autor. Es interesante ver aquellos diseñadores con lenguajes creativos consolidados que logran traspasar el soporte particular de las piezas para lograr aplicar su sello a una amplia gama de bienes. Empresas como Tramando, Y es Agua, Mucha Tela, Juana de Arco, Nadie Zlotogora, Pesqueira, Florece y Oxdans, son prueba de ello.

Adentrándonos en las familias de productos, las firmas que sólo producen prendas representan un $25 \%$, por lo que el resto de los diseñadores -75\%- producen además otros artículos que completan sus colecciones. Indagando en ese $75 \%$, podemos ver que un rubro importante lo representan los accesorios siendo las carteras/bolsos el ítem dominante -38\%-; le sigue la producción de bijouterie -20 \%-; y el calzado con un $10 \%$. La producción de otros objetos (mobiliarios y/o elementos decorativos para el hogar y/o objetos artísticos) que amplían la familia de productos, representan un $17 \%$ (INTI, 2010).

El desarrollo de colecciones a partir de familias de productos diversificadas es una de las principales estrategias comerciales utilizadas por las firmas de autor, representando muchas veces un porcentaje alto de sus ingresos. La necesidad de captar distintos segmentos del mercado a través de una variedad de productos está íntimamente relacionada al desarrollo progresivo de públicos consumidores de diseño de autor. En este sentido, la incorporación de productos diferenciados a los hábitos de consumo -dominados por la masividad del mercado- es un proceso lento pero en constante crecimiento. Las características de ese proceso varían en cada mercado local -a nivel ciudad- y se vincula inexorablemente a otras variables sociales, culturales y económicas.

\section{Impacto económico del diseño de indumentaria autor}

El impacto de la Industria Creativa del diseño de indumentaria de autor en la economía nacional es un hecho perceptible para todos pero que recientemente está comenzando a ser mensurado desde el punto de vista productivo a través de la Encuesta Económica Nacional de Diseño de Indumentaria de Autor 2010 realizada por el Observatorio de Tendencias de INTI Textiles y Fundación Pro Tejer. En este sentido, la importancia de acceder a datos confiables para poder 
obtener diagnósticos certeros adquiere más relevancia cuando se intenta elaborar políticas de apoyo al sector desde las instituciones públicas y privadas.

Siguiendo con éste análisis, podemos saber que el segmento factura $\$ 250.000 .000$ aproximadamente por año -datos correspondientes a 2009-, teniendo como base exclusivamente la producción y comercialización de prendas. Si a eso sumáramos los ingresos generados -no medido aún- sobre el total de la familia de productos que la mayoría de estas empresas analizadas producen además de prendas -habría que considerar calzado, accesorios, marroquinería y objetos- y comercializan, la facturación de la población de esas firmas seguramente se duplicaría. El fuerte impulso e interés que genera este segmento de riesgo, se traduce en el volumen de indumentaria producido anualmente que asciende aproximadamente a 615.000 prendas, incluyendo los rubros hombre y mujer.

Analizando en profundidad la capacidad de producción del segmento a través de la cantidad de prendas generadas por temporada (307.500 aprox.), podemos establecer que el $45 \%$ de los emprendimientos producen menos de 450 prendas, seguidos por un $25 \%$ que produce entre 450 y 900 prendas. A medida que subimos en la escala de producción e ingresamos a aquellas empresas que producen diseño de autor en un mayor volumen, los porcentajes de cantidad de empresas empiezan a disminuir. El $11 \%$ de las empresas produce entre 901 y 2000 prendas por temporada; un $5 \%$ entre 2001 y 4000; un $9 \%$ entre 4001 y 6000; y sólo un $5 \%$ produce más de 6000 prendas por temporada (INTI, 2010).

El volumen de producción de una empresa fluctúa entre el deseo -no todas tienen intención de generar volumen ya que hay firmas que se especializan en producir pocas cantidades de prendas haciendo de la exclusividad una estrategia comercial- y las posibilidades reales que esa empresa tiene en caso de querer incrementar la producción. En éste último caso, entran en juego tanto condicionantes de recursos -financiación, insumos, mano de obra-como así también las limitaciones que tienen ciertos diseños de poder reproducirse en grandes cantidades -prendas con trabajo intensivo artesanal dificultan este objetivo.

Continuando con el análisis de la oferta podemos saber que -cruzando los datos del volumen total producido por temporada en relación a lo producido por las distintas categorías- el segmento de empresas que producen más de $6000 \mathrm{p} / \mathrm{t}$-un $5 \%$ de la población- producen el 46 $\%$ del volumen producido en la industria de indumentaria de autor; seguidas por aquellas empresas que producen entre 4001 y 6000 p/t -9 \% de la población-, que tienen una participación de $23 \%$ sobre el volumen total. Las empresas que producen entre 2001 y 4000 p/t -5 $\%$ de la población-, representan un $9 \%$ de la oferta. Esto significa que el $19 \%$ de las empresas producen el $78 \%$ de la oferta total de diseño de autor.

Ahondando en las firmas de menor escala, con menos de 2000 p/t se encuentra el $81 \%$ de la población total de empresas de diseño de indumentaria de autor, sin embargo participan en menos del $25 \%$ de la oferta: quienes producen entre 901 y 2000 p/t $-11 \%$ de la poblaciónproducen el $9 \%$ del volumen total; quienes producen entre 451 y $900 \mathrm{p} / \mathrm{t}-\mathrm{el} 25 \%$ de la población- producen el $9 \%$ del volumen total; y el grupo más numeroso de empresas quienes producen menos de 450 prendas -representan el $44 \%$ de la población total- sólo producen el $4 \%$ del volumen total de prendas producidas (INTI, 2010).

Como dijimos anteriormente, el aumento del volumen de producción representa un gran desafío para las empresas de diseño de indumentaria de autor por las características intrínsecas de los bienes generados. Estas prendas contienen valor agregado a partir de un desarrollo que 
involucra tanto los aspectos creativos como productivos. Y si bien los lenguajes de diseño de cada uno de los diseñadores varían enormemente, en líneas generales las posibilidades de producción se dirimen en la decisión de incorporar más o menos elementos complejos a nivel constructivo y/o de detalles en las terminaciones, lo que repercute en el diálogo entre estrategias industriales y artesanales. En este punto, la necesidad de piezas innovadoras debe lidiar con las posibilidades técnicas tanto propias como de terceros - proveedores de bienes y servicios-, como así también de los costos que conllevan los distintos desarrollos del proceso productivo. Quienes tienen el interés por lograr mayores volúmenes de prendas sólo lo pueden lograr optimizando procesos y recursos. En este último punto, las empresas líderes del mercado se convierten en referentes a nivel de estrategias de producción porque logran cantidades considerables sin perder de vista el concepto de su marca.

Otro dato fundamental para medir el impacto económico refiere a la cantidad de empleo que genera ésta Industria Creativa. Actualmente las micro, pequeñas y medianas empresas dedicadas a la producción de diseño de indumentaria de autor, emplean en forma directa -a través de los estudios de diseño, talleres de producción y puntos de ventas, propios- aproximadamente a 760 personas. Y es importante saber que, en base a las expectativas favorables en el mediano plazo, estas firmas planean incorporar aproximadamente un $86 \%$ más de personal a sus plantas estables de trabajadores, lo que nos permite proyectar el crecimiento sostenido en el tiempo y buenas perspectivas del sector (período 2010 -2011).

Indirectamente, a través de la contratación de proveedores de servicios de producción -como talleres de confección, de corte, de estampación, de acabados industriales, tintorerías y manufacturas artesanales- las firmas de diseño indumentaria de autor dan trabajo en forma sostenida a cerca de 2.500 personas.

Estos números indican que el diseño de autor emplea en total a 3.260 personas aprox.; entonces, en todo el proceso productivo el $23 \%$ de los trabajadores son empleados directos de las firmas, el resto $-77 \%$ - son ocupados a través del proceso de tercerización de la producción, lo que daría una idea de la dimensión que adquiere en el presente el diseño (INTI, 2010).

A estos números faltaría agregar aquellas personas que trabajan en los canales de comercialización del diseño de indumentaria de autor que son propiedad de terceros (franchising y multimarcas, principalmente), y que tienen una importancia considerable en el mercado.

\section{Micro y Pequeñas Empresas de Diseño de Indumentaria de Autor}

Elaborar un diagnóstico productivo de las Industrias Creativas en Argentina requiere hacer hincapié en conocer tanto el tamaño como la naturaleza de las empresas de las cuales estamos hablando para poder avanzar con mayor precisión en la elaboración y aplicación de acciones de apoyo desde todos los sectores.

Como señalamos antes, el segmento de diseño de indumentaria de autor tiene una facturación anual (correspondiente a 2009) aproximada de $\$ 250.000 .000$. Teniendo en cuenta la facturación anual de las empresas y tomando como referencia la categorización de Micro y PYMES -actualizada en agosto de 2010- que utiliza la Subsecretaría de la Pequeña y Mediana Empresa y Desarrollo Regional del Ministerio de Producción de la República Argentina, podemos observar que el 80\% de los emprendimientos responde a la categoría de Micro Empresa (facturación 
hasta \$ 1.800.000). El restante 20\% se incluye dentro de la categoría Pequeña Empresa (facturación entre \$1.800.000 y \$10.300.000) (INTI, 2010).

Asimismo, tomando como variable la cantidad de trabajadores directos, buscando clasificar la empresas de acuerdo a las categoría micro, pequeñas y medianas empresas en base al criterio utilizado en otros países para definir Micro-PYMES, en el universo de los emprendimientos de diseño de indumentaria de autor en Argentina el $93 \%$ de las firmas pueden ser consideradas como micro empresas (menos de 10 empleados), y el $7 \%$ como pequeñas empresas (entre 11 y 50 empleados). De ese $93 \%$ de micro empresas, el $20 \%$ corresponde a emprendimientos compuestos por un trabajador/emprendedor. Para el $100 \%$ del universo de las empresas el emprendimiento es una forma de autoempleo para el diseñador erigiéndolo, de esta manera, como trabajador y, a la vez, como empresario.

Analizando el trabajo indirecto que las empresas de diseño de autor realizan a través de la tercerización -mencionado en párrafos anteriores- encontramos que el $18 \%$ de las firmas emplean al menos 1 persona más en su proceso productivo, mientras que el $52 \%$ ocupa entre 2 y 10 personas. Las empresas que emplean indirectamente entre 11 y 50 trabajadores en su producción representan un $24 \%$. Los porcentajes comienzan a ser significativamente menores cuando se trata del rango $51 / 100$ personas, siendo un $4 \%$, y finalmente, las firmas que ocupan indirectamente más de 100 personas son un $2 \%$.

Como ya indicamos, el 77 \% de las personas ocupadas por la producción de diseño de indumentaria de autor son en forma indirecta a través de proveedores de servicios de producción. Esto adquiere sentido cuando se analizan los indicadores referidos a talleres de confección y estampación, dos de los principales servicios requeridos por la indumentaria. Aquí encontramos que el $49 \%$ de las firmas tiene talleres de confección propios, mientras que el otro $51 \%$ debe recurrir a talleres externos. Esta diferencia es aún mayor para en el caso de los talleres de estampación -con la salvedad que no todos los diseñadores recurren a la estampación-pero significativamente sólo el $36 \%$ cuenta con talleres propios, el restante 64 \% recurre o debería recurrir en un futuro a talleres de estampación externos.

En referencia a los capitales para la actividad productiva comenzada -ya que la población considerada son micro y PYMES que se encuentran en actividad- e indagando en sus canales habituales de financiamiento el $39 \%$ afirmó que logra financiarse a partir de la reinversión de utilidades, lo que significa, que consiguen desarrollar en forma sustentable su actividad en el tiempo. El 22 $\%$ declaró lograr financiar su actividad a partir de los planes de pagos acordados con sus proveedores a 30, 60 o 90 días, en la adquisición de bienes y servicios. El $15 \%$ sostiene financiar su empresa a partir del aporte externo, esto es, de la inversión de capital de terceros. Sólo el $10 \%$ dijo acudir a créditos y servicios financieros públicos y/o privados. Respecto de quienes utilizan ahorros propios el $6 \%$ eligió esta opción. En tanto otro $7 \%$ declaró otros canales habituales como el financiamiento familiar o la venta por adelantado de productos. Finalmente, el $2 \%$ afirmó recurrir a subsidios públicos para lograr financiar su actividad (INTI, 2010).

Como veremos más adelante, la problemática de la escasa financiación disponible en el mercado domina el mapa de los obstáculos que, según los diseñadores, entorpecen el crecimiento de las empresas. Esto se ve corroborado por el escaso número de empresas que recurren al crédito $(10 \%)$. Este tema es complejo y su solución puede darse a partir de un correcto diagnóstico de las Industrias Creativas, implicando un esfuerzo de todas las partes por comprender las distintas visiones. Entre los obstáculos para acceder a los servicios financieros hay que distin- 
guir entre aquellas empresas que podrían aplicar a servicios para PYMES, donde las exigencias implican planes de negocios sólidos (muchas veces los diseñadores devenidos en empresarios no conocen de la importancia de la panificación empresarial), garantías y tasas de interés elevadas para el mediano y largo plazo. La oferta de créditos a tasas razonables aún es un tema no resuelto en la economía nacional. Por otro lado, los obstáculos que tienen las microempresas para acceder a financiamiento tiene que ver con que, por un lado, intentan aplicar herramientas destinadas a PYMES cuando en realidad no encuadran dentro de la población objetiva de esos créditos y, por otro lado, hay condicionantes endógenos de muchos de estos emprendimientos para acceder en el corto plazo al financiamiento, como ser el proceso de formalización de la microempresa -en los aspectos tributarios y laborales- que implica una tarea que lleva tiempo por la complejidad y altos costos. Las líneas de microcréditos desarrolladas por actores sociales que tienen como público a los microemprendedores puede ser una estrategia viable para acompañar el desarrollo inicial de estas microempresas.

De esta manera, podemos hacernos una idea del universo que compone las empresas de diseño de autor a la vez que nos permite vincularla a la cadena de la Agro Industria de Indumentaria y Textil. En este sentido, la red de micro, pequeñas y medianas empresas que están involucradas detrás del diseño y elaboración de una prenda nos permite visualizar los puntos de contactos actuales y potenciales. La sustentabilidad económica de las firmas de autor debe estar asociada a una óptima inserción en dicha cadena productiva pudiendo establecer puentes de colaboración entre los distintos rubros.

\section{Canales de difusión y comercialización del diseño de indumentaria de autor.}

En cuanto a las estrategias de difusión y comercialización utilizadas por las empresas, éstas adquieren rasgos particulares cuando se busca posicionar al diseño de indumentaria de autor en los mercados tradicionales. Esas particulares características residen en que los productos no son simple mercancías, sino que están asociadas a un discurso personal de su autor traducido por un lenguaje de diseño particular, son prendas cargadas de significado que expresan una identidad. Como tal, este valor agregado de diferenciación lograda a través de un trabajo intensivo hace que las piezas deban insertarse en mercados aún muy poco explorados, caracterizados por condiciones de demandas impredecibles, satisfaciendo gustos cambiantes y heterogéneos. Las firmas de autor asumen los segmentos dentro de la indumentaria que presentan un alto grado de riesgo e innovación, debiendo lidiar además con la dinámica acelerada de la moda por temporadas. Sumado a esto, la falta de formación de públicos consumidores -en muchas de las ciudades del interior- hace aún más difícil la tarea. Por ello, el conocimiento intuitivo marca la pauta de las estrategias de difusión y comercialización.

En el universo explorado de esta industria creativa, sólo el $44 \%$ de los diseñadores cuentan con tiendas propias a la calle donde comercializan sus prendas pudiendo diseñar, además, los retails de acuerdo a sus pautas estéticas y comunicativas, influyendo además en la formación del personal. El restante $56 \%$ carece de esa posibilidad.

El sistema de comercialización a través de tiendas multimarcas de terceros domina ampliamente el mercado de diseño de autor y es uno de los canales elegidos por el $84 \%$ de las firmas, pudiendo de esta manera ampliar y diversificar la presencia de sus prendas en circuitos de 
diseño de distintas ciudades, con un bajo costo, aunque dependiendo de la visión estratégica de los propietarios de las tiendas.

Respecto de las nuevas posibilidades que brinda la tecnología, pocos diseñadores -sólo el 22 $\%$ - han explorado el sistema de ventas a través de tiendas on line (WEB y Facebook, principalmente). Éste recurso comercial es señalado como uno de los canales de ventas más prometedores en el mediano plazo por los analistas de mercados, lo cual adquiere relevancia para aquellas firmas que producen lejos de los grandes centros urbanos, lo cual permite preveer un fuerte desarrollo en el futuro a la vez que establecer redes de contactos estables.

De acuerdo a datos aportados por el evento Por la Calle, Circuitos de Diseño-acción de difusión federal llevada adelante por el Observatorio de Tendencias de INTI Textiles y Fundación Pro Tejer- los circuitos (zonas geográficas claramente delimitadas dentro de las ciudades) comerciales de diseño más consolidados muestran mayor presencia de locales propios de firmas de diseño de autor, como es el caso de Palermo (83\%) y Recoleta (72\%), ambos en la ciudad de Buenos Aires, mientras que la existencia de tiendas multimarcas disminuye en forma significativa. Contrariamente los números se invierten en circuito en vías de consolidación. San Telmo (38\%) en la ciudad de Buenos Aires, Rosario (27\%) en la provincia de Santa Fe, y Córdoba capital $(23 \%)$, siguen en el segmento de circuitos comerciales donde hay considerable presencia de locales propios de diseñadores pero donde predominan tiendas multimarcas. Luego continúan dos circuitos de diseño emergentes que son Mendoza capital (20\%) y San Miguel de Tucumán (13\%).

La estrategia de tiendas multimarcas constituye la segunda opción deseada por las empresas, ya que éstas privilegian los locales propios como punto de ventas ideales donde exhibir sus colecciones dentro de un marco comunicativo integrado bajo un concepto de marca. Sin embargo, la generación de importante públicos consumidores capaces adquirir prendas de autor de forma regular justificando comercialmente la instalación de puntos de ventas propios es un aspecto difícil a sortear que requiere trabajar en el largo plazo dirigiendo los esfuerzos principalmente en la comunicación.

Asimismo, las tiendas multimarcas han probado su amplia efectividad para abrirse espacio en los mercados locales ya que la posibilidad de editar y complementar las colecciones de varias firmas de acuerdo a las características del consumidor otorga una ventaja comparativa por su flexibilidad. En muchas ciudades este tipo de tiendas son las que abren paso a diseñadores que luego deciden abrir sus propias tiendas, como así también posibilitan la presencia en el mercado local de diseñadores que producen en otras ciudades.

Otro signo para medir el grado de desarrollo de un circuito de diseño -que a la vez nos referencia a un contexto social y cultural próximo- se relaciona con la cantidad de diseñadores locales (que producen y comercializan en la ciudad analizada donde se encuentra el circuito) y diseñadores externos (que producen en otras ciudades). Esto indica la oferta de producción propia que hay en cada ciudad. Los 3 circuitos de diseño de la ciudad de Buenos Aires -Palermo (98\%), Recoleta (98\%) y San Telmo (96\%) - tienen una clara presencia de empresas que comercializan y a la vez producen en el territorio. La ciudad de Córdoba (66\%) muestra también un predominio de diseñadores locales, seguida por San Miguel de Tucumán (58\%). La ciudad de Rosario (43\%), en Santa Fe, exhibe una menor presencia producción local; seguida de cerca por Mendoza (41\%), donde el predominio de producción de diseño de otras ciudades domina la oferta. La mayor o menor presencia de diseñadores locales se relaciona con factores 
contextuales antes mencionados -educativos, sociales, culturales, económico- pero también demuestra el mayor o menor grado de receptividad al diseño local presente en esa ciudad, lo que repercute en la identidad del circuito.

La exploración de canales de ventas en el exterior es uno de los mayores atractivos -al menos idealmente- para los diseñadores de autor que buscan posicionar sus productos en nuevos mercados. Del total de firmas analizadas el $27 \%$ exporta parte de sus producciones. En términos de destinos, de ese porcentaje de firmas exportadoras el $35 \%$ lo hace a países de Latinoamérica -México y Uruguay, en primer lugar, y luego Perú, Brasil, Chile, Costa Rica, Colombia y Bolivia-; el 28 \% exporta a Europa -España, en primer término, seguido de cerca por Italia, y luego Francia y Holanda-; el 16 \% exporta a Asia -siendo Japón el destino dominante, aunque Corea del Sur también figura entre las plazas- las exportaciones a Países Árabes significa un $9 \%$; al igual que las ventas a USA, también con un $9 \%$; finalmente Rusia comienza a ser un destino para el diseño de autor con un $3 \%$.

Siguiendo con el análisis de ese $27 \%$ de firmas exportadoras, podemos saber que el $73 \%$ de las firmas exporta no más de un $20 \%$ de su producción total; y un $7 \%$ exporta entre un 21/40 $\%$. Esto significa que el $80 \%$ de las firmas de autor vuelcan al mercado interno más del $80 \%$ de su producción anual. Los diseñadores que más productos exportan -más de la mitad de su colección- representan un $20 \%$, y de ese porcentaje sólo $7 \%$ exporta más del $80 \%$ de su producción, siendo entonces, los mercados internacionales su prioridad (INTI, 2010).

\section{Las problemáticas según las empresas de indumentaria de autor}

El último punto importante a considerar son los obstáculos que las propias empresas consideran que dificultan su desarrollo. Los diseñadores/empresarios encuentran que a la hora de gestionar sus empresas las dificultades en la obtención de financiamiento (17\%) es el principal problema. En los párrafos anteriores estuvimos hablando sobre la problemática que se produce por el defasaje entre la naturaleza de las empresas y las herramientas disponibles, sin embargo la dificultad de acceder a tasas de interés razonables es uno de los grandes cuellos de botella para la producción. La falta de difusión de su marca y productos $(13 \%)$ y el proceso inflacionario (13\%), también representan obstáculos aunque muy distintos, porque mientras el primero corresponde a las estrategias de comunicación propias de una empresa, el segundo tiene que ver con el contexto económico. Otro de los problemas identificados es la escasez de personal calificado (11\%), lo que demuestra las complicaciones que muchas veces encuentran los bienes diferenciados para se realizados, aspecto que se complica aún más cuando estas empresas carecen de recursos para formar en el tiempo su propia mano de obra calificada. La caída de rentabilidad (10\%) también es un escollo a sortear; aquí debiéramos profundizar en cómo se determinan los márgenes de rentabilidad en el universo de estas empresas y su pertinencia al contexto económico. La falta de conocimiento de canales comerciales por los cuales distribuir sus productos $(9 \%)$ es otro de los obstáculos elegidos que responden a factores endógenos. Con el mismo porcentaje figura el ítem de caída de las ventas (9\%) registradas en el último período (2009) coincidente con una desaceleración del crecimiento de la economía nacional. La falta de insumos (9\%) para poder producir adecuadamente es algo de lo que hablamos anteriormente y que, en gran medida, podemos asignar a la oferta limitada de bienes y servicios a la 
que pueden acceder con costos competitivos la empresas del segmento. El retraso en los cobros a clientes (cadena de pago) (4\%) también figura así como el aumento de los salarios (4\%) de los trabajadores de la empresa. Finalmente, la falta de conocimiento de los procesos productivos (3\%) hace su aparición en último lugar como un obstáculo para el desarrollo de su actividad. ${ }^{16}$ Comparativamente, si sumáramos aquellos aspectos que hacen al contexto económico dentro del que insertan los emprendimientos tendríamos que los factores exógenos a la empresa representan el $49 \%$ de los obstáculos para el desarrollo de la actividad. Asimismo, si sumáramos aquellos factores endógenos a la gestión de los emprendimientos tendríamos más del $50 \%$. Esto es revelador porque exhibe el profundo trabajo que hay que hacer tanto para aportar capacitación y transferencia de conocimientos a las empresas -para que puedan aplicar mejores estrategias de diseño, producción y comercialización- como para lograr establecer canales de colaboración entre los emprendimientos y los proveedores de bienes y servicios productivos y financieros -logrando una industria creativa integrada plenamente a la cadena productiva de indumentaria y textil.

\section{Desafíos del diseño de indumentaria de autor}

La Industria Creativa del Diseño de Autor es estratégica para cualquier país. Por un lado, entraña un considerable valor económico ya que genera empleo, incorpora emprendedores al sistema productivo, agrega valor a nivel local y dinamiza el mercado, permitiendo de ésta manera garantizar la diversidad de la trama productiva. Por otro lado, la importancia cultural es indiscutible en tanto que los bienes que ese segmento produce revisten un alto contenido simbólico que permite comunicar una fuerte identidad social, actualizando y proyectando el patrimonio cultural de una comunidad. El fortalecimiento de este sector implica un trabajo a largo plazo tanto de los emprendedores como de las instituciones que tienen como fin colaborar en su desarrollo.

Los diseñadores al frente de sus empresas deben considerar como tarea fundamental la constante capacitación para poder llevar de manera eficiente su doble rol: como creativo y como empresario. Asimismo es importante que la permanente experimentación que sus procesos creativos conllevan -y que implican un alto valor de diferenciación de los bienes- vaya acompañada de un mejoramiento de los procedimientos productivos para alcanzar criterios de calidad que faciliten la posterior distribución de los mismos.

En cuanto a políticas públicas, los aspectos críticos que presentan muchos de los emprendimientos en términos de gestión, producción, difusión y comercialización, deben ser contemplados a partir de un diagnóstico certero sobre la situación actual de las unidades para luego proponer soluciones viables. Estas respuestas deben contemplar principalmente tanto la generación de contenidos -recordemos lo novedoso del fenómeno- y transferencia de conocimientos a través de capacitaciones; como la creación de herramientas productivas y financieras perdurables a largo plazo que contemplen la naturaleza de las empresas (micro y pequeñas, principalmente). La investigación de mercados nacionales e internacionales y la apertura de nuevos públicos consumidores, también debe estar contemplada en políticas de promoción al diseño.

Finalmente, sólo la colaboración estrecha entre empresas, Estado e instituciones educativas, puede garantizar la eficiencia de las acciones futuras tendientes a consolidar la Industria Creativa de Diseño de Indumentaria de Autor en Argentina. 


\section{Referencias Bibliográficas}

Gertnet, A. (2008). Mapa de Diseño Argentino, Observatorio de Tendencias INTI. (2007-10). Fundación Pro Tejer.

INTI (2010). Encuesta Económica Nacional de Diseño de Indumentaria de Autor 2010, Observatorio de Tendencias INTI. (2010). Fundación Pro Tejer, ediciones INTI.

Las Cosas del Quehacer, debates en torno al diseño de indumentaria en Argentina, Centro Cultural España Córdoba - Observatorio de Tendencias INTI. (2010). Fundación Pro Tejer, ediciones CCEC.

Mapa de Diseño Argentino, Observatorio de Tendencias INTI (2007-10). Fundación Pro Tejer. Por la Calle, Circuitos de Diseño, Observatorio de Tendencias INTI. (2008-10). Fundación Pro Tejer. www.xlacalle.com

\section{Bibliografía}

Gertnet, A. (2008). Mapa de Diseño Argentino, Observatorio de Tendencias INTI. (2007-10). Fundación Pro Tejer.

INTI (2010). Encuesta Económica Nacional de Diseño de Indumentaria de Autor 2010, Observatorio de Tendencias INTI. (2010). Fundación Pro Tejer, ediciones INTI.

Las Cosas del Quehacer, debates en torno al diseño de indumentaria en Argentina, Centro Cultural España Córdoba - Observatorio de Tendencias INTI. (2010). Fundación Pro Tejer, ediciones CCEC.

Mapa de Diseño Argentino, Observatorio de Tendencias INTI (2007-10). Fundación Pro Tejer. Por la Calle, Circuitos de Diseño, Observatorio de Tendencias INTI. (2008-10). Fundación Pro Tejer. www.xlacalle.com

Summary: A productive and cultural diagnosis of the Creative Industries of Author Fashion Design in Argentina, to 10 years of its emergence, will allow us to measure the real impact of this sector as well as to identify the main challenges to obtain economic and social sustainability in the long term.

Key words: author fashion design - creativity - culture - Design - diagnosis - industry - local development - sustainability.

Resumo: Um diagnóstico produtivo e cultural das Indústrias Criativas de Design de Indumentária de Autor na Argentina, a 10 años do seu surgimento, permitirá medir o real impacto deste setor à vez que identificar os principáis desafios para lograr a sustentabilidade econômica e social no longo prazo.

Palavras chave: criatividade - cultura - desenvolvimento local - Design - diagnóstico - Indumentária de Autor - Indústria - sustentabilidade. 